\title{
Respostas das geleiras do Monte Melimoyu, Chile, às tendências mais secas e quentes da Patagônia Norte nas últimas décadas
}

\section{Response of Mount Melimoyu glaciers, Chile, to dryer and warming climatic trends for Northern Patagonian region in last decades}

\author{
Filipe Daros Idalino* $₫($ ID, Kátia Kellem da Rosa $\bowtie$ (D), Jefferson Cardia Simões $₫$ (iD \\ Centro Polar e Climático, Instituto de Geociências, Universidade Federal do Rio Grande do Sul, \\ Porto Alegre, Rio Grande do Sul, Brasil \\ E-mails: katia.rosa@ufrgs.br (KKR); jefferson.simoes@ufrgs.br (JCS) \\ *E-mail para correspondência: filipe.daros.idalino@gmail.com
}

Recebido (Received): 26/07/2019

Aceito (Accepted):16/03/2020

\begin{abstract}
Resumo: Neste trabalho foram investigadas variações de área nas geleiras no Monte Melimoyu $\left(44^{\circ} \mathrm{S}, 72^{\circ} \mathrm{O}\right)$ no período de 1970-2017, e as possíveis relações com a variabilidade climática entre 1950 e 2017. Dados ópticos do Sentinel-2A, de elevação do Modelo Digital de Elevação (MDE) ASTER-GDEM v.2, e de área das geleiras do inventário GLIMS foram utilizados na análise em SIG. As séries temporais de precipitação do Explorador Climático - Chile, e dados de reanálise de temperatura da universidade de Delaware foram utilizados para a análise climática. A área das geleiras diminuiu de 80,97 km² em 1970 para $52,14 \mathrm{~km}^{2} \mathrm{em}$ 2017, representando 35,6\% de área, com destaques para a retração de geleiras no Oeste, Sudoeste, Norte e Nordeste, com perdas entre $65 \%$ e $44 \%$ de área total, e variações de elevação da linha de frente entre $74 \mathrm{~m}$ e $570 \mathrm{~m}$. A precipitação anual média aproximada foi de $2.359 \mathrm{~mm}$ no período de 1950 a 2017 e mostra tendência de diminuição de aproximadamente $-18 \mathrm{~mm} / \mathrm{ano}$, enquanto que os dados de temperatura mostram média anual de $9,87^{\circ} \mathrm{C}$ e uma tendência contínua de aumento na região, de $0,04^{\circ} \mathrm{C}$ entre 1948 e 2017 . Os contrastes entre as geleiras em perda de área e variação de elevação da linha de frente estão relacionados às diferenças de área e geomorfometria de cada geleira. Essas geleiras são semelhantes em tamanho, altitude e declividade do setor frontal, e as variações identificadas podem estar relacionadas às tendências mais quentes e secas para o período analisado.
\end{abstract}

Palavras-chave: Variação glacial; Patagônia Norte chilena; Análise multitemporal; Mudanças climáticas.

\begin{abstract}
In this paper were investigated area variations in glaciers on Mount Melimoyu $\left(44^{\circ} S, 72^{\circ} \mathrm{W}\right)$ in the period of 1970-2017, and possible relationships with the climate variability between 1950 and 2017. Optical data from Sentinel-2A, and elevation from Digital Elevation Model (DEM) ASTER-GDEM v.2 and area data from GLIMS inventory were used in GIS analyse. The temporal series of annual precipitation data by Explorador Climático - Chile, and temperature data of reanalysis from the University of Delaware were utilised for climate analysis. The glaciers area reduced from $80.97 \mathrm{~km}^{2}$ in 1970 to $52.14 \mathrm{~km}^{2}$ in 2017, equivalent to a $35.6 \%$ reduction in area, especially as for the glaciers located in the West, Southwest, North, and Northeast sectors, with losses between $65 \%$ and $44 \%$ of total area, and front-line elevation variations between $74 \mathrm{~m}$ and $570 \mathrm{~m}$. The mean precipitation was $2539 \mathrm{~mm}$ in the period 1950-2017 and is evidenced a tendency to decrease precipitation of approximately $-18 \mathrm{~mm}$ per year. The temperature data show an annual average of $9.87^{\circ} \mathrm{C}$ and a continuous trend of increase in the region of $0.04^{\circ} \mathrm{C}$ between 1948 and 2017. The contrasts between glaciers in loss of area and variation of front line elevation are related to differences in area and geomorphometry of each glacier. These glaciers are similar in size, elevation and slope of the front sector, and the variations identified can be related to warm, and dryer trends for the region in the analysed period.
\end{abstract}

Keywords: Glacier fluctuations; Northern Chilean Patagonia; Multitemporal analysis; Climate changes. 


\section{Introdução}

Na região Chilena e Argentina dos Andes são observadas mudanças nas geleiras, como balanço de massa negativo, com a retração glacial e diminuição de volume (RIGNOT et al., 2003; COUDRAIN et al., 2005; CASASSA et al., 2007; RABATEL et al., 2013; PELLICCIOTTI et al., 2014; MERNILD et al., 2015; IPCC, 2016). As geleiras na Patagônia Norte estão retraindo (RIGNOT et al., 2003; ANIYA, 2007; MÖLLER et al., 2007; MASIOKAS et al., 2008; GARREAUD et al., 2013; MALMROS et al., 2016). É relevante a investigação das mudanças nas geleiras, pois estas trazem consequências para os recursos hídricos, riscos naturais e flutuações do nível dos mares (MEIER, 1984; DYURGEROV e MEIER, 2000; HAEBERLI et al., 2000; KASER, 2001; ARENDT et al., 2002; HUGGEL et al., 2002; BISHOP et al., 2004; KARGEL et al., 2005; CASASSA et al., 2007; ROCHA e GIERING, 2017).

A exemplo disso, Masiokas et al. (2008) atribuíram à recessão de geleiras da região Norte da Patagônia, no setor entre $39^{\circ} \mathrm{S}$ e $43^{\circ} \mathrm{S}$, uma tendência para condições mais secas e mais quentes detectadas durante o período 1912-2002. Schaefer et al. (2013) usaram um modelo para avaliar o balanço de massa superficial do campo de gelo da Patagônia Norte no passado, evidenciando que a acumulação aumentou de 1990 para 2011 em relação a 1975-1990, enquanto as perdas de massa dobraram em 2000-2009, em comparação com 19752000.

Bertrand et al. (2012) também apontaram que as flutuações da geleira Gualas, no Campo de Gelo da Patagônia Norte, foram principalmente controladas por mudanças na precipitação. González (2004) registrou o aumento da elevação da linha de equilíbrio nas geleiras nas últimas décadas na Região dos Lagos $\left(39^{\circ}\right.$ a $42^{\circ} \mathrm{S}$ ), e relacionou ao aquecimento troposférico e à redução das precipitações regionais, por diminuir a área de acumulação destas.

Embora o clima seja o fator preponderante no regime térmico também há influência da topografia, que por sua vez também influencia no padrão local de precipitação (SANCHES, 2013). Com isso, a declividade no setor frontal das geleiras pode ser um fator determinante no processo de ablação, o que pode influenciar em uma maior velocidade de deslizamento basal (PATERSON, 1999).

O Monte Melimoyu, localizado a $44^{\circ} 5^{\prime} \mathrm{S}-72^{\circ} 51^{\prime} \mathrm{W}$, na região norte da Patagônia Chilena, corresponde a um estrato vulcânico com aproximadamente 9,65 quilômetros $(\mathrm{km})$ de diâmetro, com $52,14 \mathrm{~km}^{2}$ de área glacial em 2017 (IDALINO et al., 2019) e uma cratera do tipo caldeira coberta de gelo (Figura 1). Está situado a $21 \mathrm{~km}$ de distância da costa leste do Pacífico Sul, entre os rios Palena e Moraleda, onde o degelo escoa para os fiordes em contato com o oceano. A Patagônia Norte, assim como a XI região administrativa do Chile onde está inserido o Monte Melimoyu (a região de Aisén), é caracterizada por uma paisagem complexa com muitos fiordes e lagos (Figura 1), que foram formados por erosão laminar glacial do manto de gelo da Patagônia durante o Quaternário (VANDEKERKHOVE, 2014).

O Monte Melimoyu apresenta, a frente geleira de número 16 (Figura 1), uma feição deposicional chamada de Geleira Rochosa, que são feições reconhecidas pela estrutura característica e pela cobertura superficial de detritos, e que são comuns em geleiras de montanha, por consequência da própria capacidade erosiva do corpo de gelo (FERRANDO, 2017). Essa cobertura pode ser caracterizada como mantos detríticos e pode estar relacionada a deposição morâinica, podendo influenciar de diversas maneiras a dinâmica de uma geleira, onde sua dinâmica e possíveis mecanismos de alimentação-fusão estão claramente relacionados à interação entre precipitação de neve, o aumento de aporte radiativo, o aquecimento resultante e o papel das diferentes espessuras da cobertura de detritos (FERRANDO, 2017).

As geleiras do Monte Melimoyu experimentaram uma perda de área de 35,61\% do total de $80,97 \mathrm{~km}^{2} \mathrm{em}$ 1970, apresentando $52,14 \mathrm{~km}^{2}$ em 2017. Mostraram também, uma contínua tendência à retração no período de análise, e as tendências de diminuição da precipitação média anual podem explicar as recentes variações nestas geleiras (IDALINO et al., 2019). No entanto, pouco ainda se sabe sobre todas as variáveis que podem influenciar nesses processos de alterações no ambiente, tal como a variabilidade climática e a variação na elevação da linha de frente nas geleiras do Monte Melimoyu.

Este artigo objetiva investigar a relação da variação da elevação de linha de frente da área das geleiras no Monte Melimoyu com a variabilidade da precipitação e temperatura média anual no período 1950-2017. O artigo analisa os contrastes entre as variações de elevação da linha de frente das geleiras a partir das diferenças de declividade que acompanham seu recuo. 


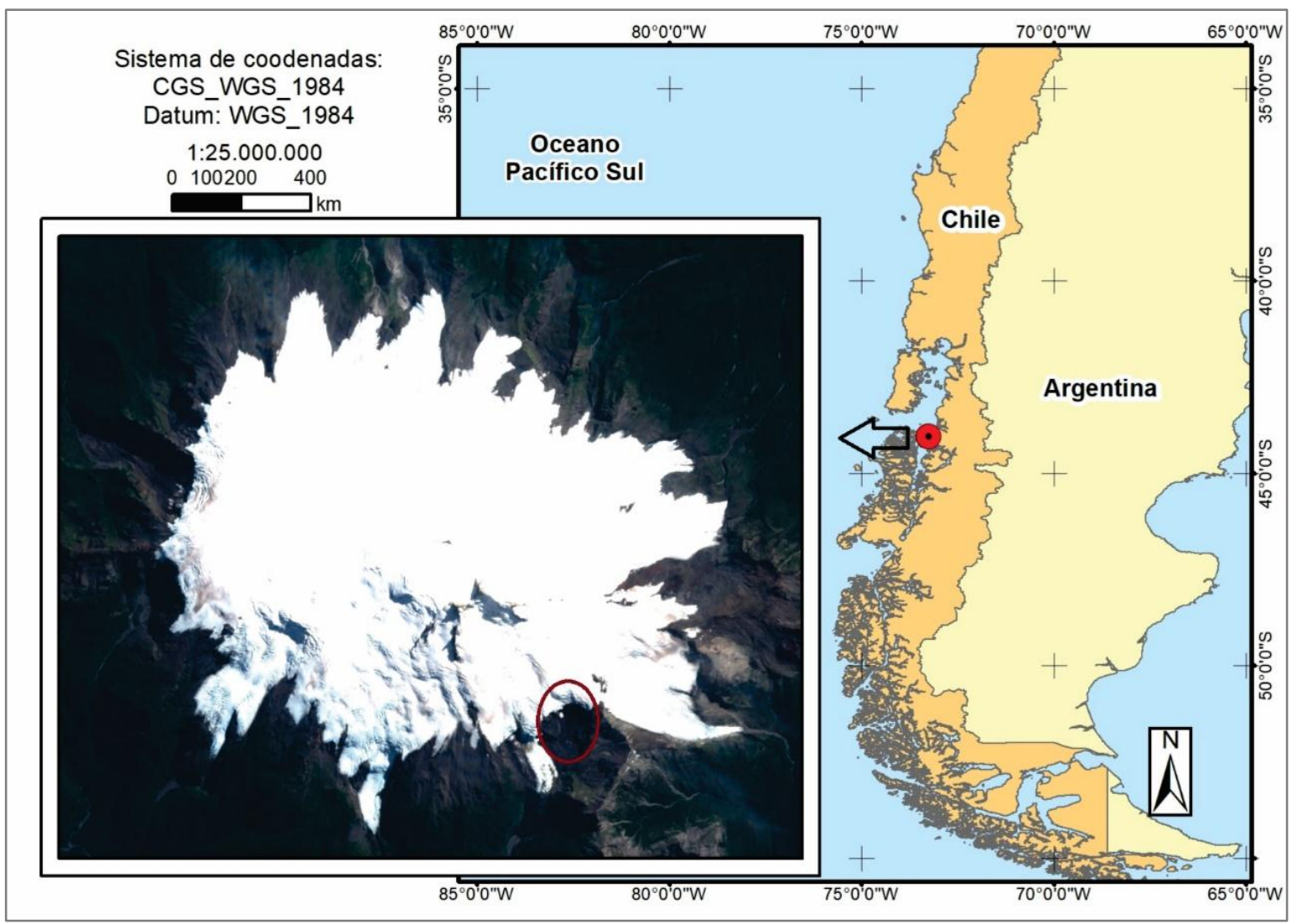

Figura 1: Localização do Monte Melimoyu. Geleira rochosa demarcada com a elipse em vermelho. Imagem do Sentinel-2A com RGB (4-3-2) cor-verdadeira. Fonte: elaboração própria com dados do Sentinel-2 (USGS-NASA) e do IBGE.

\section{Materiais e métodos}

Para a execução da metodologia foram utilizados o Modelo Digital de Elevação (MDE) ASTER-GDEM v.2 (2011), uma cena do Sentinel-2A - MSI, LC1 (17/03/2017) e dados de área das geleiras em formato shapefile do Global Land Ice Measurements from Space (GLIMS, 2015) para os anos de 1970, 1986 e 2000, e dados de Idalino et al. (2019) para o ano de 2017 (Tabela 1). Para as análises meteorológicas foram utilizadas a série de dados de precipitação média anual da região das estações de La Junta ( $43^{\circ} 58^{\prime} \mathrm{S}$ $\left.72^{\circ} 25^{\prime} \mathrm{O}\right)$ e Puerto Aysén Ad. $\left(45^{\circ} 23^{\prime} \mathrm{S}-72^{\circ} 39^{\prime} \mathrm{O}\right)$ entre 1950 e 2017 (Tabela 1), e a série de dados de reanálise de Delaware das médias anuais de temperatura superficial do ar, no período de 1948-2017.

A detecção da variação da elevação da linha de frente foi estimada a partir dos valores das cotas de elevação mínima das geleiras (em 1970 e em 2017) através da análise das linhas de frente de 1970, 1986, 2000 e 2017 e do ASTER-GDEM v2, o qual foi projetado para o sistema de projeção UTM, fuso 18S Datum WGS 1984. O MDE, em formato raster, foi processado no software ArcGis, versão 10.3.1 (ESRI®), para extrair as informações geomorfométricas das geleiras, como a elevação, a declividade e a orientação.

Tabela 1: Dados utilizados no trabalho.

\begin{tabular}{cccc}
\hline Dados & $\begin{array}{c}\text { Escala / resolução } \\
\text { espacial / medidas }\end{array}$ & Data / período & Fonte \\
\hline ASTER-GDEM v.2 & 30 metros & 2011 & USGS \\
Sentinel-2A, MSI, LC1 & 10 metros & $17 / 03 / 2017$ & USGS \\
Área das geleiras em 1970, & 30 metros & 2015 & glims.org/rgi \\
1986 e 2000 & 10 metros & 2018 & Idalino et al. 2019 \\
Área de geleiras em 2017 & Milímetros (mm) por ano & $1970-2017$ & $(\mathrm{CR})^{2}$ - Chile \\
Precipitação & $\begin{array}{c}\text { Médias anuais em graus } \\
\text { Celsius }\left({ }^{\circ} \mathrm{C}\right)\end{array}$ & $1970-2017$ & Delaware - GHCN2 \\
Temperatura & & &
\end{tabular}


O MDE foi classificado com intervalos de 200 metros (m) de altitude, entre $175 \mathrm{~m}$ (cota mínima de elevação) e 2.399 m (cota máxima de elevação) para caracterização dos percentuais envolvidos em cada classe de elevação do Monte Melimoyu. A declividade da superfície foi extraída da classificação do MDE, aplicando a ferramenta de análise espacial Slope, do Surface, ao arquivo raster, obtendo intervalos expressos em graus entre $0-3^{\circ}, 3-8^{\circ}, 8-20^{\circ}, 20-45^{\circ}, 45-70^{\circ}$ e $>70^{\circ}$.

Após o processamento e extração das informações, foi realizado o recorte do MDE com os dados de área das geleiras (em formato shapefile) do GLIMS para o ano de 1970 e de Idalino et al. (2019) para o ano de 2017. Os dados representam os limites das 16 geleiras (delimitados pelo GLIMS), bem como a definição das linhas de frente. O recorte foi aplicado com as áreas totais em 1970 e em 2017 de cada geleira para os respectivos períodos.

A reclassificação dos arquivos raster foi realizada com a ferramenta de análise 3D Reclassify, do Raster Reclass. Esse procedimento permitiu a tabulação dos valores de elevação e declividade para conversão dos polígonos por meio da conversão Raster to Polygon. Nesse formato, foi possível extrair o valor da área total em $\mathrm{km}^{2}$ para cada classe de elevação e declividade das geleiras, permitindo o cálculo de porcentagem (\%) de área por classe.

\section{Resultados e discussões}

\subsection{Variações geomorfométricas e dados meteorológicos}

A área total coberta por gelo do Monte Melimoyu em 1970 era de $80,97 \mathrm{~km}^{2}$, e de $52,14 \mathrm{~km}^{2}$ em 2017 (IDALINO et al., 2019). As geleiras com maior perda percentual de área total foram as de número 10, 12, 4 , 11 e 15 , com $64,94 \%, 57,63 \%, 50 \%, 49,86 \%$ e 44,27\% respectivamente; as de menor perda percentual de área foram as de número $14,2,7,8$ e 5 , com $28,71 \%, 28 \%, 25,37 \%, 23,08 \%$ e $19,72 \%$, respectivamente. As demais geleiras $(3,9,6,13,1$ e 16) apresentaram $39,11 \%, 37,77 \%, 36,89 \%, 34,28 \%, 32,45 \%$ e $31,65 \%$ (Tabela 2).

Tabela 2: Percentual da perda de área total das geleiras, entre 1970-2017.

\begin{tabular}{ccccc}
\hline Geleira & $\begin{array}{c}\text { Área }\left(\mathbf{k m}^{\mathbf{2}}\right) \\
\mathbf{1 9 7 0}\end{array}$ & $\begin{array}{c}\text { Área }\left(\mathbf{k m}^{\mathbf{2}}\right) \\
\mathbf{2 0 1 7}\end{array}$ & $\begin{array}{c}\text { Área total }(\mathbf{\%}) \\
\text { restante entre } \\
\mathbf{1 9 7 0 - 2 0 1 7}\end{array}$ & $\begin{array}{c}\text { Perda total }(\%) \\
\text { entre } \mathbf{1 9 7 0 - 2 0 1 7}\end{array}$ \\
\hline $\mathbf{1}$ & 6,07 & 4,1 & 67,54 & 32,45 \\
$\mathbf{2}$ & 0,5 & 0,36 & 72 & 28 \\
$\mathbf{3}$ & 3,63 & 2,21 & 60,88 & 39,11 \\
$\mathbf{4}$ & 0,54 & 0,27 & 50 & 50 \\
$\mathbf{5}$ & 2,89 & 2,32 & 80,27 & 19,72 \\
$\mathbf{6}$ & 13,12 & 8,28 & 63,1 & 36,89 \\
$\mathbf{7}$ & 4,02 & 3 & 74,62 & 25,37 \\
$\mathbf{8}$ & 8,36 & 6,43 & 76,91 & 23,08 \\
$\mathbf{9}$ & 4,95 & 3,08 & 62,22 & 37,77 \\
$\mathbf{1 0}$ & 4,25 & 1,49 & 35,05 & 64,94 \\
$\mathbf{1 1}$ & 3,73 & 1,87 & 50,13 & 49,86 \\
$\mathbf{1 2}$ & 2,62 & 1,11 & 42,36 & 57,63 \\
$\mathbf{1 3}$ & 9,45 & 6,21 & 65,71 & 34,28 \\
$\mathbf{1 4}$ & 2,02 & 1,44 & 71,28 & 28,71 \\
$\mathbf{1 5}$ & 1,31 & 0,73 & 55,72 & 44,27 \\
$\mathbf{1 6}$ & 13,52 & 9,24 & 68,34 & 31,65 \\
\hline
\end{tabular}

O comportamento de retração apresentado vai ao encontro dos resultados encontrados em trabalhos realizados para outras áreas da Patagônia, como Rignot et al. (2003), Aniya (2007), Möller et al. (2007), Masiokas et al. (2008), Lopez et al. (2010), Garreaud et al. (2013) e Schaefer et al. (2013).

Em relação ao total de área coberta por gelo em 1970, a geleira 13 mostrou área nos setores representados pelas classes de 175-200 m, 201-400 m (Figura 2) e na classe de 401-600 m, juntamente com as geleiras 6, 7 e 16 (Figura 2 e Tabela 3). Em vista disso, as mesmas geleiras apresentaram os mais altos valores de retração no início do período de análise (IDALINO et al., 2019), que mostra que fator da elevação da linha de frente teve influencia direta na perda de área dessas geleiras (Tabela 3 e Figuras 2 e 3). 
Tabela 3: Classes de elevação com menor área coberta por geleiras em 1970.

\begin{tabular}{ccc}
\hline Classe & Área $^{\mathbf{k m}^{2}}$ & $\mathbf{\%}$ \\
\hline $175-200$ & 0,03 & 0,03 \\
$201-400$ & 0,52 & 0,64 \\
$401-600$ & 1,9 & 2,34 \\
Total: & $\mathbf{2 , 4 5}$ & $\mathbf{3 , 0 1}$ \\
\hline \multicolumn{2}{c}{ Área total em $1970: 80,97 \mathrm{~km}^{\mathbf{2}}$}
\end{tabular}

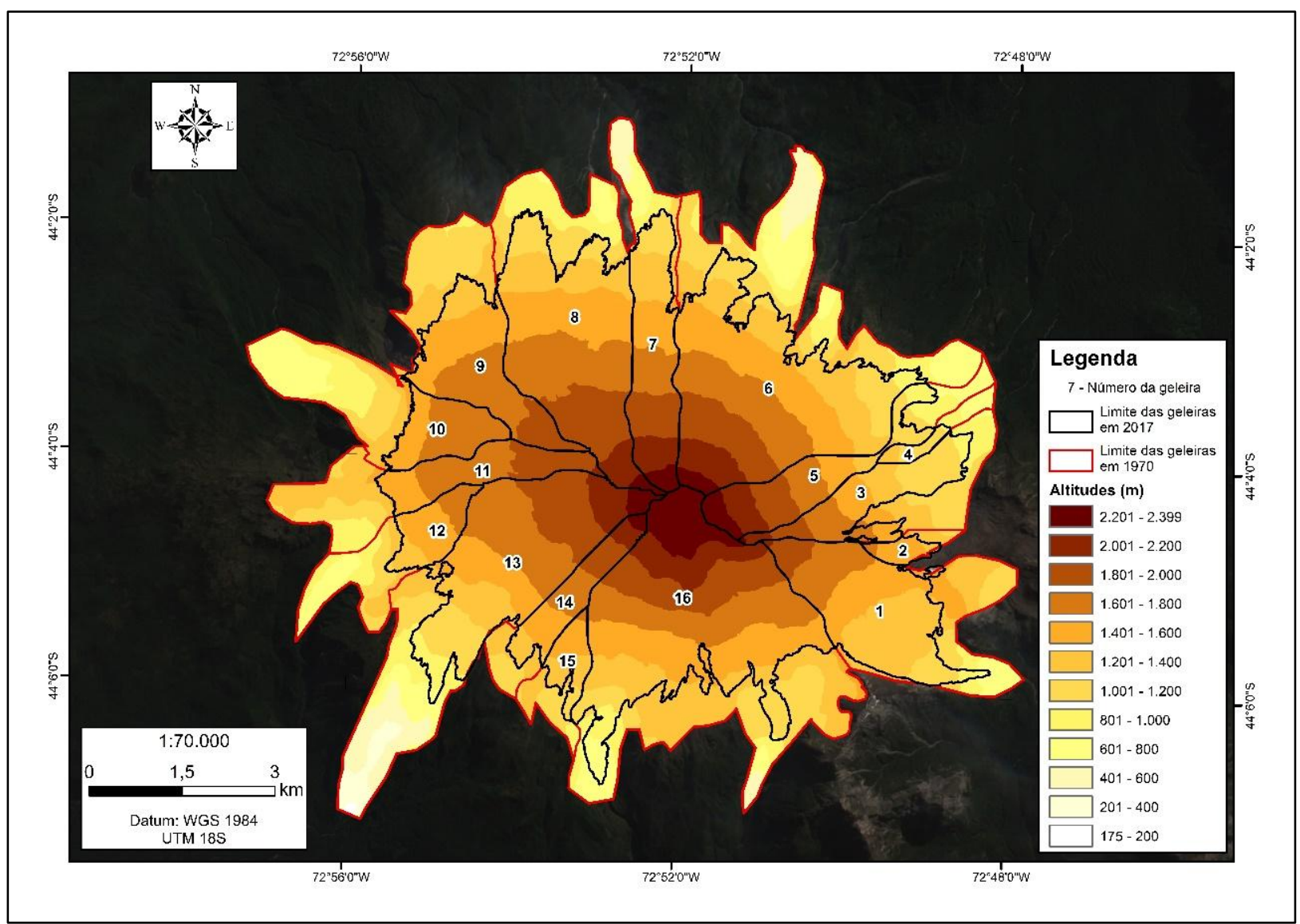

Figura 2: Geleiras e caracterização das classes de elevação do Monte Melimoyu em 1970 e 2017. Imagem do Sentinel-2A com RGB (4-3-2) cor-verdadeira. Fonte: elaboração própria com dados do ASTER-GDEM v. 2 e do Sentinel-2 (USGS-NASA).

As linhas de frente mostraram variações de elevação ao longo de todo o período de análise (Tabela 3 e Figuras 2 e 3). As maiores variações da cota mínima estão associadas às geleiras 10, 11, 13, 7 e 12, com um aumento de 74 m, 570 m, 528 m, $477 \mathrm{~m}$ e 468 m, respectivamente (Figura 3), e as menores variações foram apresentadas pelas geleiras 5, 14, 2, 3 e 1, com um aumento de $192 \mathrm{~m}, 178 \mathrm{~m}, 146 \mathrm{~m}, 128 \mathrm{~m}$ e $98 \mathrm{~m}$, respectivamente (Figura 3).

As altas variações das cotas mínimas de elevação são produto do processo de retração frontal experimentado por todas as geleiras entre 1970-2017 (IDALINO et al., 2019). O processo resultou na perda de área total nessas classes, e em 2017, o Monte Melimoyu não apresenta mais áreas de geleiras nas classes de elevações de 175-200 m, 201-400 m e 401-600 m (Figuras 2 e 3).

Em se tratando de temperatura superficial do ar, os dados de reanálise de Delawere mostraram uma média $9,87^{\circ} \mathrm{C}$ para o período de $1948-2017$, com tendência positiva de aumento nos dados para o período (Figura 4). Verifica-se um maior aumento nos valores de temperatura média na década de 1950 e 1970, com $6,65^{\circ} \mathrm{C}$ em $1953,9,13^{\circ} \mathrm{C}$ em $1958,9,65^{\circ} \mathrm{C}$ em 1971 e $11,13^{\circ} \mathrm{C}$ em 1979 , o que representou a maior variação nas médias de temperatura de toda a série histórica (Figura 4). No início da década de 1980, as médias voltaram a apresentar médias mais baixas, chegando a $9,88^{\circ} \mathrm{C}$ no ano de 1994 (Figura 4). Também houve grande variabilidade nos anos seguintes, chegando até $10,97^{\circ} \mathrm{C}$ em $1987,11,15^{\circ} \mathrm{C} \mathrm{em} 1989,10,13^{\circ} \mathrm{C}$ em 1991 e $11,11^{\circ} \mathrm{C}$ em 1998 , o ano mais quente da década de 1990 . Os valores de média mais baixa e mais alta da série temporal ocorreram nos anos iniciais da década de 2000 , com $9,54^{\circ} \mathrm{C}$ em 2002 , e $11,38^{\circ} \mathrm{C}$ em 2004 (Figura 4). 


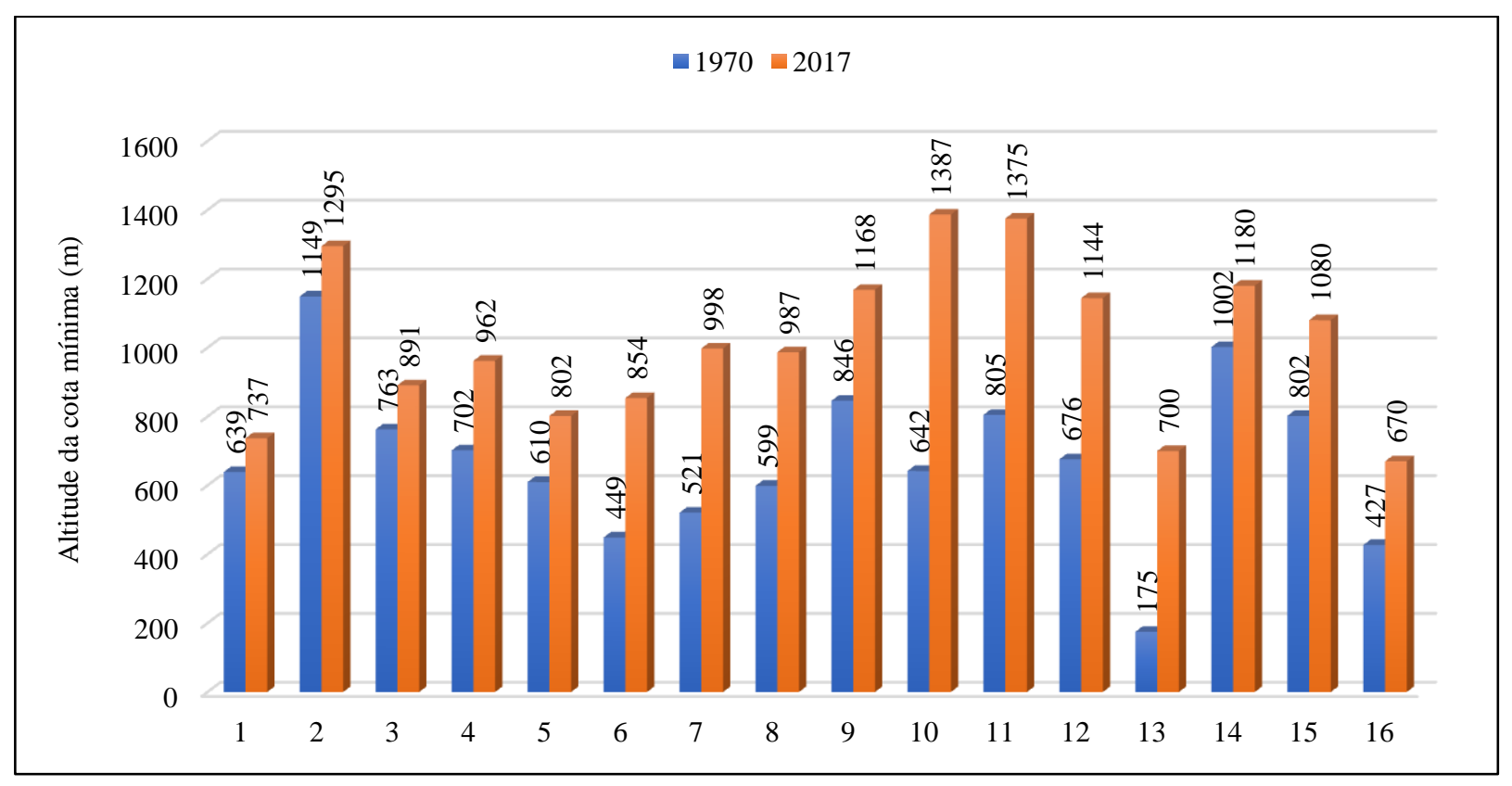

Figura 3: Variação da elevação da linha de frente das geleiras. Fonte: Elaboração própria.

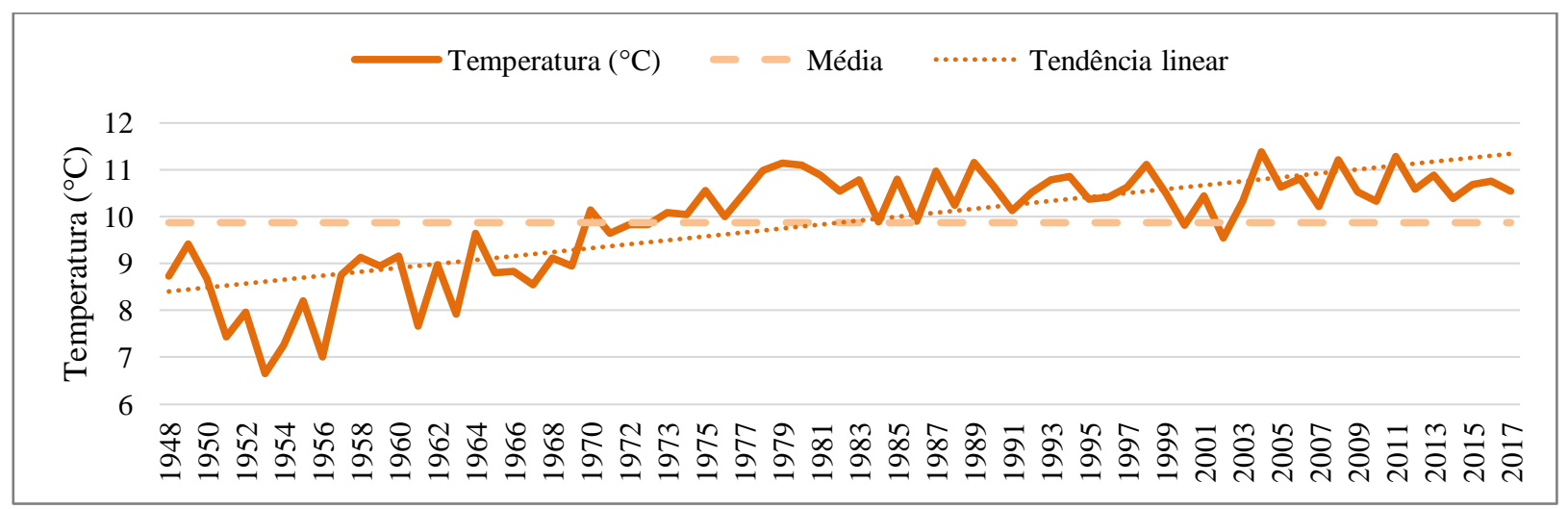

Figura 4: Gráfico de médias anuais da temperatura superficial do ar, a partir dos dados de reanálise de Delaware. Fonte: elaboração própria com dados da universidade de Delaware. Disponível em: http://climate.geog.udel.edu/. Acesso em 13/12/2018.

Em se tratando de precipitação, de acordo coma a série temporal a média anual para o período é de 2.539 milímetros $(\mathrm{mm})$. A série mostra que há uma tendência contínua de diminuição nos valores de precipitação total na região (Figura 5). Ambas as estações meteorológicas apresentam valores de precipitações médias anuais semelhantes, com uma tendência decrescente em todo o período de análise.

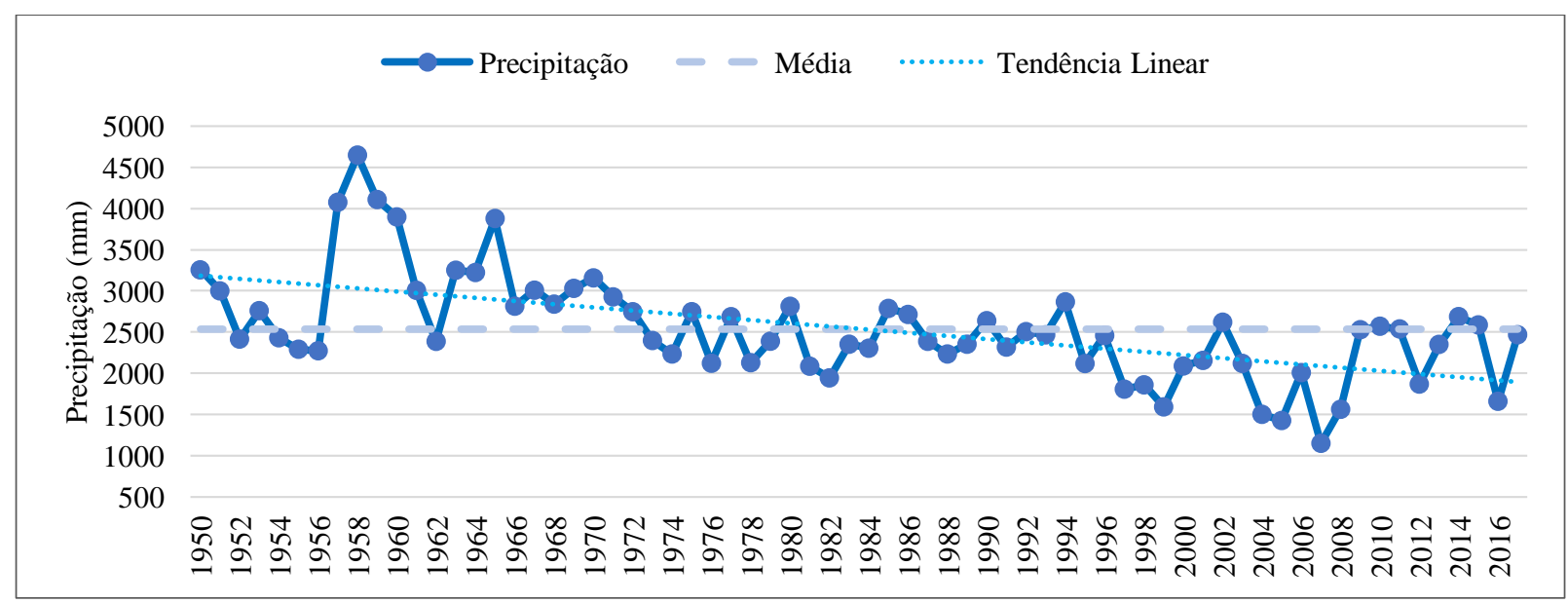

Figura 5: Gráfico da precipitação média anual com dados das estações meteorológicas de La Junta e Puerto Aysén Ad. Fonte: elaboração própria com dados do Explorador Climático $(\mathrm{CR})^{2}$. Disponível em: http://explorador.cr2.cl. Acesso em 05/04/2018. 
Observam-se baixos valores de precipitação na década de 1970, quando comparados com os valores nos anos iniciais do período, onde em 1958 obteve-se uma precipitação de $4.647 \mathrm{~mm}$, por exemplo, e início da década de 1980, chegando a $2.234 \mathrm{~mm}$ em 1974 e $1.944 \mathrm{~mm}$ no ano de 1982 (Figura 5). Um aumento significativo de precipitação foi verificado no ano de $1985 \mathrm{com} 2.787 \mathrm{~mm}$, e, logo após, uma redução nos anos finais da década, chegando $2.353 \mathrm{~mm}$ em 1989 (Figura 5).

As décadas de 1990 e 2000 mostram uma tendência de declínio na precipitação (a exceção dos anos de 1994 e 2002 por aumentos nos valor de precipitação, com $2.866 \mathrm{~mm}$ e $2.242 \mathrm{~mm}$, respectivamente), e os anos de 2005 e 2007 apresentaram os mais baixos valores de precipitação na região em todo o período de análise, com $1.427 \mathrm{~mm}$ e $1.151 \mathrm{~mm}$, respectivamente, configurando os anos mais secos da série (Figura 5).

Nos anos iniciais do período de análise, as geleiras mostravam menores valores de elevação nos setores frontais, bem como em áreas de maiores declividades (Figuras 2, 3 e 4). A análise da variabilidade meteorológica destas décadas iniciais do período de estudo é relevante, pois o processo de retração das geleiras foi mais acentuado no período de 1970 a 1986 (Figura 6), em resposta às condições mais quentes e secas apresentadas pelos dados meteorológicos, e a presença de gelo em locais de baixa elevação (Figuras 2, 4, 5 e 6). No período entre 1986 e 2000, o comportamento de retração das geleiras foi menos pronunciado (Figura 6), o que pode estar ligado a menor variabilidade dos dados meteorológicos (Figuras 4, 5 e 6).

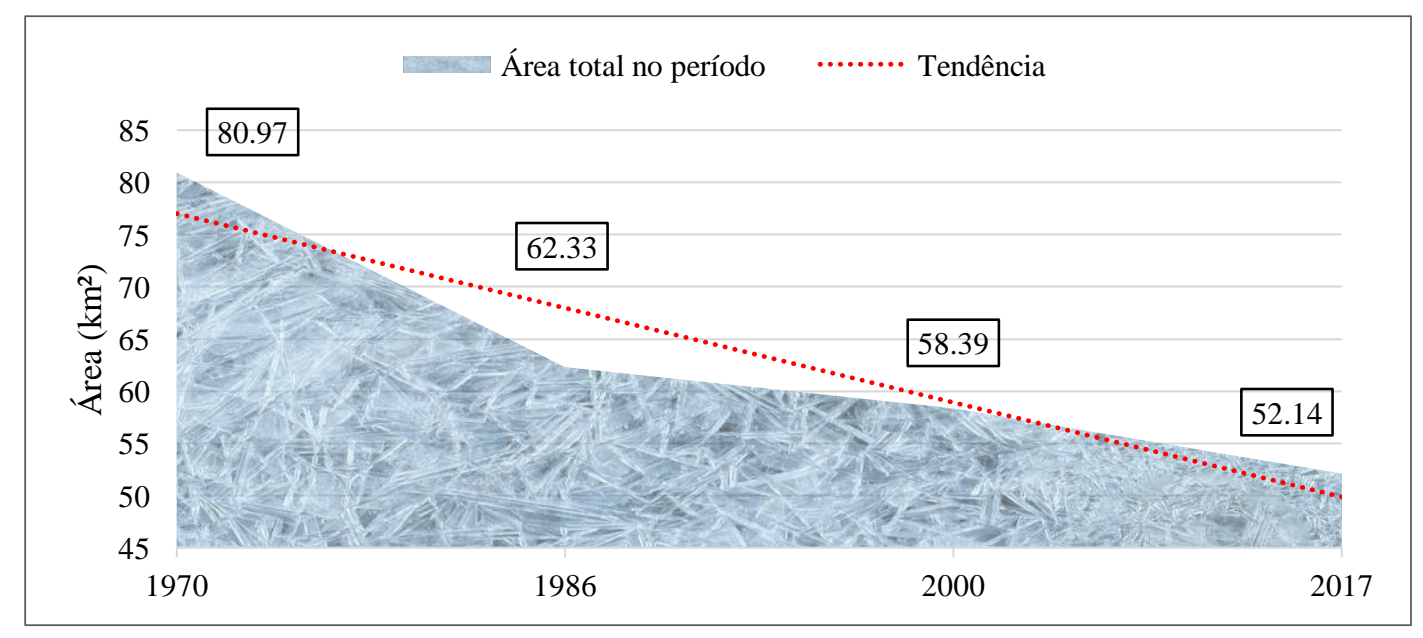

Figura 6: Gráfico de área total por período de análise. Fonte: elaboração própria com dados do GLIMS, 2015 e de Idalino et al. (2019).

Com a diminuição da altitude, a temperatura aumenta e a precipitação tende a se tornar cada vez mais líquida e a dinâmica das geleiras, incluindo o regime termal, pode ser influenciada por essa condição, causando aumento de ablação e, por consequência, retração das linhas de frente, aumento da cota mínima de elevação e diminuição da área total das geleiras (Figuras 3, 5 e 6).

Os estudos existentes na região indicam que a variável climática exerce uma das maiores influências no comportamento dessas geleiras, pois o aumento das temperaturas favorece o derretimento das camadas de gelo, forçando o processo de ablação das geleiras na Patagônia Norte e alterando o equilíbrio dos corpos de gelo (GONZÁLEZ, 2004; MASIOKAS et al., 2008; BERTRAND et al., 2012; PELLICCIOTTI et al., 2014). Com isso, verificou-se uma relação direta do comportamento de retração das geleiras com as tendências apresentadas nos dados meteorológicos, de diminuição de precipitação e aumento da temperatura. Essa relação ficou ainda mais evidente no início do período de análise (Figuras 2, 4, 5 e 6), onde se vê diferenças abruptas de temperaturas com médias mais elevadas, e quedas acentuadas nos valores de precipitação na década de 1970 se estendendo até o início da década de 1980, período em que se registrou os maiores valores de retração.

\subsection{Variação da declividade e o impacto nas geleiras}

As classes de declividade entre $8-20^{\circ}$ e $20-45^{\circ}$ (Figura 7) representam as classes com maior cobertura de área total durante todo o período de análise. Em 1970, as classes de $8-20^{\circ}$ e $20-45^{\circ}$ representavam $45,42 \%$ e $39,93 \%$ do total de $80,97 \mathrm{~km}^{2}$ de área glacial do Monte Melimoyu (Figura 7), e em 2017, essas classes somam 52,95\% e 36,75\% do total de $52,14 \mathrm{~km}^{2}$ de área glacial (Figura 7). 


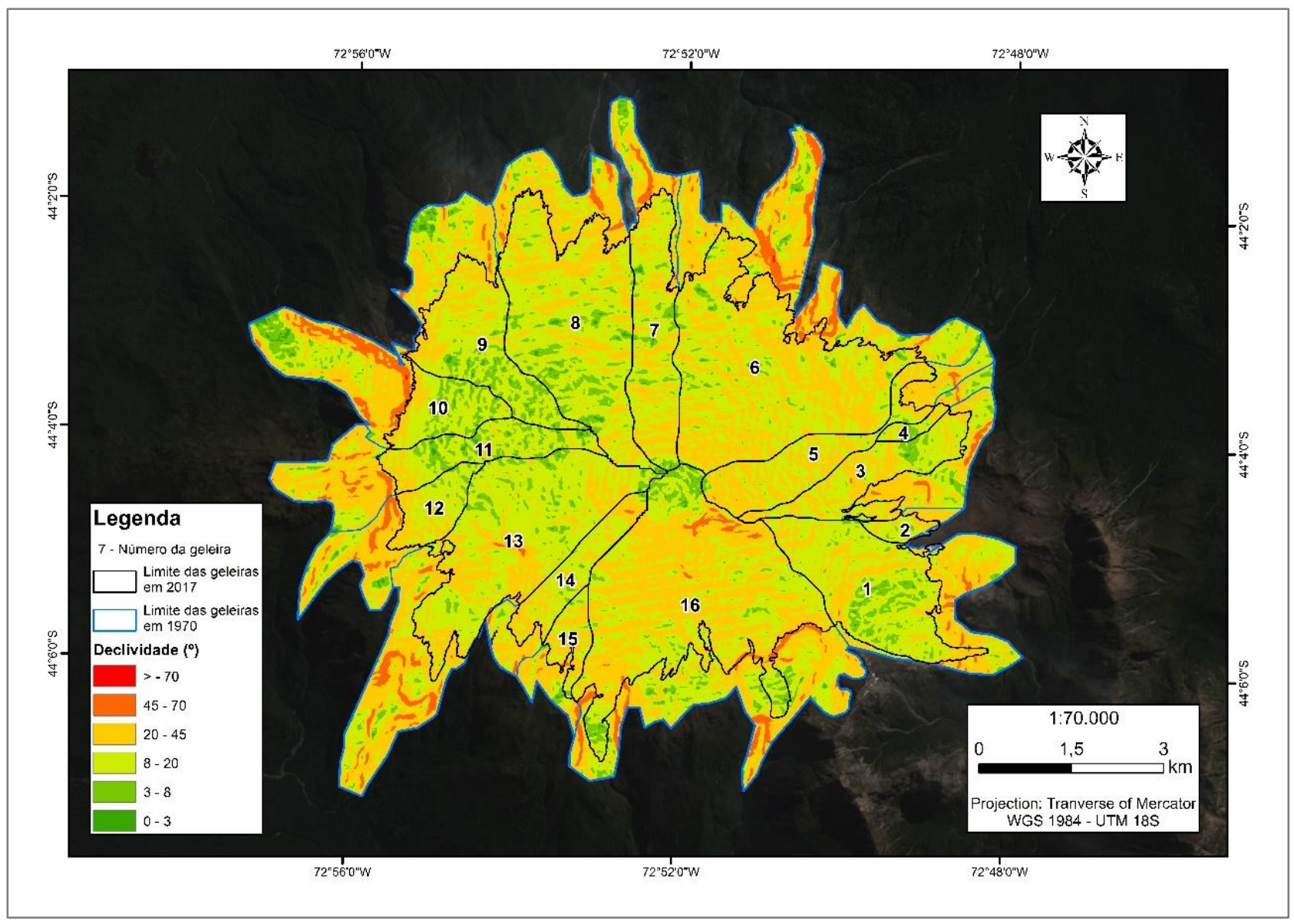

Figura 7: Declividade das geleiras no Monte Melimoyu em 1970 e 2017. Imagem do Sentinel-2A com RGB (4-3-2) cor-verdadeira. Fonte: elaboração própria com dados do ASTER-GDEM v.2 e do Sentinel-2 (USGS, NASA).

Em 1970, as classes de declividade entre $0-3^{\circ}$, de $3-8^{\circ}$, de $45-70^{\circ}$ e $>70^{\circ}$ apresentavam os menores valores de área glacial total, sendo $2,44 \mathrm{~km}^{2}, 6,19 \mathrm{~km}^{2}, 3,20 \mathrm{~km}^{2}$ e $0,014 \mathrm{~km}^{2}$, respectivamente, o que representava $3,01 \%, 7,65 \%, 3,95 \%$ e $0,017 \%$ do total de área coberta por gelo apresentado em 1970 (Figura 7 e Tabela 4). Em 2017, as classes de $0-3^{\circ}$, de $3-8^{\circ}$ e de $45-70^{\circ}$ também apresentaram as classes com menos área coberta por gelo, com $0,5 \mathrm{~km}^{2}(0,95 \%), 4,57 \mathrm{~km}^{2}(8,76 \%)$ e $0,28 \mathrm{~km}^{2}(0,55 \%)$ da área total (Figura $7 \mathrm{e}$ Tabela 4). As áreas com valores de declividade $>70^{\circ}$ em 1970 desapareceram em 2017 (Figura 7 e Tabela 4).

O contraste de perda de área total e elevação da linha de frente de algumas geleiras (como as de número 6, 10 e 13) no período mostra relação com a presença de setores de declividade máxima em seus ambientes proglaciais, formados entre 1970 a 2017 (Tabela 4). As geleiras de número 6, 7, 10 e 11 possuíam $0,009 \mathrm{~km}^{2}, 0,0013 \mathrm{~km}^{2}, 0,0027 \mathrm{~km}^{2}$ e $0,0009 \mathrm{~km}^{2}$ de área na classe de declividade $>70^{\circ}$, e em 2017 os setores frontais destas se encontram nas classes de $20-45^{\circ}$ e de $45-70^{\circ}$ (Tabela 4).

Foi observado que geleiras que possuíam altos valores de declividade frontal em 1970, também apresentaram altas taxas de retração no período analisado, como as de número $11,6,7,15,13,9$ e 8 (Figura 7). Considerando, então, a influência dos fatores declividade do setor frontal, da elevação máxima e suas dimensões em 1970, geleiras de menor área são mais suscetíveis a retração quando possuem áreas em locais de altos valores de declividade, como é o caso das geleiras de número 15, 11, 7 e 8 (Figura 7). Ao contrário dessas, as geleiras 5 e 2, com setor frontal em locais de menor declividade que as citadas anteriormente, foram as que apresentaram as menores variações de elevação frontal e também baixa perda percentual de área no período 1970-2017 (Figuras 2, 3 e 7).

Evidencia-se, então, que a magnitude da resposta de cada geleira na área de estudo às forçantes pode ser modulada pelos fatores topográficos, como a declividade e elevação no setor frontal. Em relação a isso, Howat et al. (2002) destacam que relação entre as características morfométricas e as variações de área é uma importante interação a ser observada em análises de geleiras. Aniya e Enomoto (1986) também ressaltam a relevância de entender a tipologia e topografia da zona de ablação no estudo dos diferentes comportamentos das geleiras na Patagônia Norte. 
Tabela 4. Declividades mínimas, médias e máximas em 1970 e 2017.

\begin{tabular}{ccccccccccc}
\hline Geleira & \multicolumn{3}{c}{ Declividade em 1970 } & \multicolumn{3}{c}{ Declividade em 2017 } & $\begin{array}{c}\text { Variação da } \\
\text { mínima }\end{array}$ & $\begin{array}{c}\text { Variação da } \\
\text { média }\end{array}$ & $\begin{array}{c}\text { Variação da } \\
\text { máxima }\end{array}$ \\
\hline Id & Mínima & Média & Máxima & Mínima & Média & Máxima & $\mathbf{1 9 7 0 - 2 0 1 7}$ & $\mathbf{1 9 7 0 - 2 0 1 7}$ & $\mathbf{1 9 7 0 - 2 0 1 7}$ \\
\hline $\mathbf{1}$ & 0,53 & 18,78 & 61,33 & 0,53 & 15,66 & 49,73 & 0 & $-3,12$ & $-11,6$ \\
$\mathbf{2}$ & 2,25 & 20,53 & 43,81 & 2,25 & 18,2 & 39,41 & 0 & $-2,33$ & $-4,4$ \\
$\mathbf{3}$ & 0,37 & 20,39 & 60,05 & 0,37 & 18,45 & 48,32 & 0 & $-1,94$ & $-11,73$ \\
$\mathbf{4}$ & 0,37 & 16,62 & 56,14 & 0,37 & 14,33 & 38,34 & 0 & $-2,29$ & $-17,8$ \\
$\mathbf{5}$ & 0,75 & 21,62 & 53,09 & 0,75 & 20,98 & 49,01 & 0 & $-0,64$ & $-4,08$ \\
$\mathbf{6}$ & 0,37 & 22,91 & 72,83 & 0,37 & 19,99 & 49,89 & 0 & $-2,92$ & $-22,94$ \\
$\mathbf{7}$ & 0,1 & 19,54 & 70,97 & 0,37 & 17,22 & 46,73 & $-0,27$ & $-2,32$ & $-24,24$ \\
$\mathbf{8}$ & 0,1 & 18,07 & 64,04 & 0,1 & 16,01 & 50,65 & 0 & $-2,06$ & $-13,39$ \\
$\mathbf{9}$ & 0,1 & 17,35 & 66,92 & 0,75 & 15,05 & 50,87 & $-0,65$ & $-2,3$ & $-16,05$ \\
$\mathbf{1 0}$ & 0,1 & 22,14 & 72,13 & 0,37 & 13,83 & 68,73 & $-0,27$ & $-8,31$ & $-3,4$ \\
$\mathbf{1 1}$ & 0 & 20,25 & 70,39 & 0,1 & 12,32 & 33,13 & $-0,1$ & $-7,93$ & $-37,26$ \\
$\mathbf{1 2}$ & 0,37 & 23,71 & 68,29 & 1,12 & 17,01 & 39,79 & $-0,75$ & $-6,7$ & $-28,5$ \\
$\mathbf{1 3}$ & 0,37 & 20,45 & 68,69 & 0,53 & 16,54 & 54,8 & $-0,16$ & $-3,91$ & $-13,89$ \\
$\mathbf{1 4}$ & 0,84 & 20,12 & 59,65 & 0,84 & 18,9 & 52,69 & 0 & $-1,22$ & $-6,96$ \\
$\mathbf{1 5}$ & 0,1 & 22,55 & 61,41 & 1,19 & 20,06 & 47,86 & $-1,09$ & $-2,49$ & $-13,55$ \\
$\mathbf{1 6}$ & 0,37 & 22,08 & 67,64 & 0,53 & 21,2 & 64,37 & $-0,16$ & $-0,88$ & $-3,27$ \\
\hline
\end{tabular}

Segundo Nesje e Dahl (2000), o efeito de uma determinada flutuação climática no balanço de massa da geleira depende da distribuição área-altitude da geleira. Quanto a isso, a análise comparativa entre a variação das elevações de linhas de frente e das classes de elevação mostra que as geleiras 10 e 13 são algumas das que apresentaram maior retração frontal no período de 1970-2017 (IDALINO et al., 2019), o que mostra que, além das condições mais secas e quentes experimentadas no período, este processo pode estar diretamente ligado aos baixos valores de altitude apresentados no setor frontal no início do período de análise.

Como a temperatura atmosférica decresce com a altitude, a neve depositada nas maiores altitudes tende a possuir menores temperaturas do que as depositadas em altitudes mais baixas (OERLEMANS, 2001). De certa forma, se a temperatura aumenta com a diminuição da altitude e a precipitação tende a se tornar cada vez mais líquida, a dinâmica das geleiras pode ser influenciada por essa condição climática, causando aumento de ablação e, por consequência, retração das linhas de frente.

Ressalta-se que as diferenças existentes nos climas locais, nos tamanhos e da declividade podem explicar o comportamento diferente de cada geleira, assim como denotam Nesje (1992), Casassa et al. (1997), Harrison e Winchester (1998) e, especificamente para a área de estudo, Idalino et al. (2019). As avalanchas, por exemplo, são especialmente condicionadas a terrenos íngremes. Este é um processo responsável pelo transporte de camadas espessas de neve e gelo dessas áreas (PELLICCIOTTI et al., 2014; KUHN et al., 1999), e a presença de área das geleiras em setores com alta declividade, principalmente iguais ou maior que $70^{\circ}$ de declividade, pode influenciar diretamente no processo de retração ocorrido nas geleiras do Monte Melimoyu.

A cobertura sedimentar também pode ser considerada com um parâmetro condicionante para as respostas das geleiras. É comum encontrar geleiras que conseguem preservar suas características de área por conta do isolamento térmico, muitas vezes relacionado com camadas de sedimentos. Segundo Ferrando (2017), algumas geleiras rochosas em regiões montanhosas podem apresentar uma cobertura de detritos muitas vezes tão uniforme que se tornam quase imperceptíveis quando próximas às encostas e outras geleiras de rochas. A cobertura sedimentar pode ter efeito de transmissor ou isolante térmico a partir de $\pm 3 \mathrm{~m}$ de espessura, dependendo da seleção granulométrica e da litologia (FERRANDO, 2017). Quanto a isso, a geleira rochosa (destacada na Figura 1), identificada por Ferrando (2017), aparentemente se encontra em um processo de isolamento térmico ocasionado pela cobertura de detritos, que deriva de uma geleira branca regenerada (FERRANDO, 2017). Contudo, a geleira rochosa identificada exerceu pouca ou nenhuma influência nos fatores analisados no trabalho, visto que a mesma, em 2017, se encontra isolada das demais geleiras. 


\section{Conclusões}

Ao analisar as médias dos dados de temperatura e precipitação no período 1950-2017 foram encontradas tendências de aumento nas médias anuais de temperatura superficial do ar e de diminuição nas médias anuais de precipitação. Os dados de área total das geleiras apresentaram uma tendência contínua de diminuição de área percentual em todo o período. Com isso, o comportamento das geleiras analisadas pode estar diretamente relacionado com as tendências de condições mais secas e quentes analisadas no período. Estes resultados vão ao encontro de outros estudos na Patagônia realizados por Masiokas et al. (2008), Schaefer et al. (2013) e Pellicciotti et al. (2014) sobre as variações das geleiras nas últimas décadas na região, e contribuem significativamente para o entendimento das variações ocorridas nessa região da criosfera.

A maior variabilidade meteorológica para os anos iniciais do período pode ser um fator chave para explicar a retração mais acentuada entre 1970 e 1986, onde os dados meteorológicos mostraram um comportamento significativo de aquecimento e de estiagem entre as décadas de 1950 e 1980. As condições menos variáveis nos dados meteorológicos das décadas seguintes, em contraste com as condições geomorfométricas de cada geleira, podem ter tido influencia direta no processo de retração dos corpos de gelo, tornando-o mais suave, porém contínuo.

Coincidindo com estes resultados, as geleiras possuíam, no início do período de análise, os mais baixos valores de elevação nos setores frontais, bem como os setores frontais em locais de alta declividade. Dessa maneira a combinação destes fatores pode explicar os contrastes entre as variações de elevação da linha de frente e as variações das áreas de cada geleira do Monte Melimoyu no período de análise. Um banco de dados de imagens, com maior detalhe temporal de mapeamento das geleiras, pode preencher as lacunas de informações sobre a variação área, de declividade do relevo, e da influência da altitude no comportamento das geleiras durante o período de análise, e até mesmo na formação de geleiras rochosas e suas características. Tal banco de dados também possibilitaria investigar outras variáveis importantes em estudos glaciológicos, como o balanço de massa e a detecção da variação de linha de neve com dados de campo, o que pode auxiliar no entendimento do comportamento das geleiras dessa região.

Os resultados indicam uma significativa perda de área das geleiras, o que demonstra que o processo de retração é uma resposta direta as tendências de condições mais secas e quentes no analisadas no período, e a análise da geomorfometria também aponta que a topografia exerce uma forte influência nos corpos de gelo, e, por isso, esses importantes parâmetros estão diretamente ligados ao processo de retração. Os resultados obtidos também possibilitaram a disponibilização de um banco de dados atualizado em SIG, contendo informações relevantes das características das geleiras dos Monte Melimoyu, e a análise dos dados também pode colaborar com monitoramentos futuros sobre as mudanças de geleiras da região.

\section{Agradecimentos}

Ao Conselho Nacional de Desenvolvimento Científico e Tecnológico (CNPq). Ao Instituto Nacional da Ciência e Tecnologia (INCT) da Criosfera e à Coordenação de Aperfeiçoamento de Pessoal de Nível Superior - CAPES (Projeto CAPES 88887.136384/2017-00). À Fundação de Amparo à Pesquisa do Estado do Rio Grande do Sul (FAPERGS). Ao Centro Polar e Climático (CPC) e ao Programa de Pós-Graduação em Geografia (POSGEA) - UFRGS.

\section{Referências}

ANIYA, M. Glacier variations of Hielo Patagónico Norte, Chile, for 1944/45-2004/05. Bulletin Glaciological Research, v. 24. p. 59-70, 2007. Disponível em: <http://web.seppyo.org/bgr/pdf/24/BGR24p59.pdf>.

ANIYA, M.; ENOMOTO, H. Glacier Variations and Their Causes in the Northern Patagonia Icefield, Chile, since 1944. Arctic and Alpine Research, v. 18, n. 3 p. 307-316, 1986. Doi: https://doi.org/10.2307/1550888.

ARENDT, A. et al. Rapid Wastage of Alaska Glaciers and their Contribution to Rising Sea Level. Science, v. 297. p. 382-386, 2002. Doi: 10.1126/science.1072497.

BERTRAND, S. et al. Precipitation as the main driver of Neoglacial fluctuations of Gualas glacier, Northern Patagonian Icefield. Climate of the Past. v. 8, p. 519-534, 2012. Doi: 10.5194/cp-8-519-2012. 
BISHOP, M. et al. Global land-ice Measurements from Space (GLIMS): Remote Sensing and GIS Investigations of the Earth's Cryosphere. Geocarto International. v. 19, n.2. p. 57-84, 2004. Doi: $10.1080 / 10106040408542307$.

CASASSA, G. et al. A century-long recession record of Glacier O'Higgins, Chilean Patagonia. Annals of Glaciology. 24. p. 106 - 110, 1997. Doi: 10.1017/s0260305500012015.

CASASSA, G. et al. Current Status of Andean Glaciers. Science Direct, Global and Planetary Change, v. 59, p. 1-9, 2007. Doi: 10.1016/j.gloplacha.2006.11.013.

COUDRAIN, A.; FRANCOU, B.; KUNDZEWICZ, Z. Glacier shrinkage in the Andes and consequences for water resources - Editorial. Hydrological Sciences Journal. 50(6). p. 925-932, 2005. Doi: 10.1623/hysj.2005.50.6.925.

DYURGEROV, M.; MEIER, M. Twentieth Century Climate Change: Evidence from Small Glaciers. Proceedings of the National Academy of Science, v.97, n.4, p. 1406-1411, 2000. Doi: 10.1073/pnas.97.4.1406.

DGA. Dirección General de Aguas, Ministerio de Obras Públicas. Gobierno de Chile. Informaciones de los dados pluviométricos. Disponível em: http://www.dga.cl/acercadeladga/Paginas/default.aspx.

ESA. U.S. Geological Survey. Sentinel-2A. 17 de março de 2017. 4349'51.39"S, 7304'24.36"W. Informações disponíveis em: < https://earthexplorer.usgs.gov/metadata/10880/1207546/>.

FERRANDO, F. Sobre la distribución de Glaciares Rocosos en Chile, análisis de la situación y reconocimiento de nuevas localizaciones. Investigaciones Geográficas, v. 54, p. 127-144, 2017. Doi: 10.5354/0719-5370.2017.48045.

GARREAUD, R. et al. Large-Scale Control on the Patagonian Climate. Journal of Climate, v.26, p. 215230, 2013. Doi: 10.1175/JCLI-D-12-00001.1.

GONZÁLEZ, F, B. Cambios climáticos en la Región de Los Lagos y respuestas recientes del Glaciar Casa Pangue (41 ${ }^{\circ}$ 's'S). Facultad de Arquitectura y Urbanismo, Escuela de Postgrado, Departamento de Geografia. Universidad de Chile, 131, 2004. Doi: 10.4067/S0034-98872004000500007.

HAEBERLI, W.; BARRY, R.; CIHLAR, J. Glacier Monitoring Within the Global Climate Observing System. Annals of Glaciology, v. 31, p.241-246, 2000. Doi: 10.3189/172756400781820192.

HARRISON, S.; WINCHESTER, V. Historical fluctuations of the Gualas and Reicher Glaciers, North Patagonian Icefield, Chile. The Holocene, v. 8, n. 4, p. 481-485, 1998. Doi: 10.1191/095968398672334459.

HOWAT, I, M.; JOUGHIN, I.; SCAMBOS, T. A. Rapid changes in ice discharge from Greenland outlet glaciers. Science, v. 315, p. 1559-1561, 2007. Doi: 10.1126/science.1138478.

HUGGEL, C. et al. Remote Sensing Based Assessment of Hazards from Glacier Lake Outbursts: A Case Study in the Swiss Alps. Canadian Geotechnical Journal, v. 39, n. 2, p. 316-330, 2002. Doi: 10.1139/t01099.

IDALINO, F, D. et al. Recent glacier variations on Mount Melimoyu (44.50'S-72 51 'W), Chilean Patagonia, using Sentinel-2 data. Geocarto International, p. 1-16. 2019. Doi: 10.1080/10106049.2018.1557262.

IPCC, 2013. Climate Change 2013: The Physical Science Basis. Contribution of Working Group I to the Fifth Assessment Report of the Intergovernmental Panel on Climate Change [STOCKER, T.F., D. QIN, G.-K. PLATTNER, M. TIGNOR, S.K. ALLEN, J. BOSCHUNG, A. NAUELS, Y. XIA, V. BEX AND P.M. MIDGLEY (eds.)]. Cambridge University Press, Cambridge, United Kingdom and New York, NY, USA, 1535 pp. Disponível em: <https://www.ipcc.ch/site/assets/uploads/2018/02/WG1AR5_all_final.pdf>.

KARGEL, J. et al. Multispectral Imaging Contributions to Global Land Ice Measurements from Space. Remote Sensing of the Environment, v. 99, p. 187-219, 2005. Doi: 10.1016/j.rse.2005.07.004. 
KASER, G. Glacier-Climate Interaction at Low Latitudes. Journal of Glaciology. 47. 195-204, 2001. Doi: $10.3189 / 172756501781832296$.

KUHN, M. et al. Measurements and models of the mass balance of hintereisferner. Geografiska Annaler. 81(4). 659-670, 1999. Doi: 10.1111/j.0435-3676.1999.00094.x.

MALMROS, J, K.; MERNILD, S, H.; WILSON, R. Glacier area changes in the central Chilean and Argentinean Andes 1955-2013/14. Journal of Glaciology, v. 62, p. 391-401, 2016. DOI: $10.1017 /$ jog.2016.43.

MASIOKAS, M. et al. 20th-century glacier recession and regional hydroclimatic changes in northwestern Patagonia. Global and Planetary Change, v. 60, p. 85-100, 2008. Doi: 10.1016/j.gloplacha.2006.07.031.

MEIER, M. Contribution of Small Glaciers to Global Sea Level. Science. 226. 1418-1421, 1984. Doi: $10.1126 /$ science.226.4681.1418.

MERNILD, S, H. et al. Mass loss and imbalance of glaciers along the Andes Cordillera to the Sub-Antarctic islands. Global and Planetary Change. 133. 109-119, 2015. Doi: 10.1016/j.gloplacha.2015.08.009.

MÖLLER, M.; SCHNEIDER, C.; KILIAN, R. Glacier change and climate forcing in recent decades at Gran Campo Nevado, southernmost Patagonia. Annal of Glaciology, 46. 136-144, 2007. DOI: $10.3189 / 172756407782871530$.

NASA/METI. U.S. Geological Survey. Advanced Spaceborn Thermal Emission Radiometric. Global Digital Elevation Model, version 2. 17 de outubro de 2011. 44 $30^{\circ} \mathrm{S}, 72^{\circ} 30^{\prime} \mathrm{W}$. Informações disponíveis em: <https://earthexplorer.usgs.gov/metadata/4220/ASTGDEMV2_0S45W073/>.

PATERSON, W, S, B. The Physics of Glaciers. Butterworth-Heinemann, third edition. 1999.

OERLEMANS, J. Extracting a Climate Signal from 169 Glacier Records. Science, v. 308, n. 5722, p. 675677, 2005. Doi: 10.1126/science.1107046.

PELLICCIOTTI, F. et al. Changes of Glaciers in the Andes of Chile and Priorities for Future Work. Science of the Total Environment, v. 493, p. 1197-1210, 2014. Doi: 10.1016/j.scitotenv.2013.10.055.

RABATEL, A. et al. Current state of glaciers in the tropical Andes: a multi-century perspective on glacier evolution and climate change. The Cryosphere. 7. 81-102, 2013. Doi: 10.5194/tc-7-81-2013.

RIGNOT, E; RIVERA, A; CASASSA, G. Contribution of the Patagonia Icefields of South America to Sea Level Rise. Science, v. 302, p. 434-437, 2003. Doi: 10.1126/science.1087393.

ROCHA, S, A.; VIDELA, GIERING, Y. Caracterización Glaciológica de Chile. Investigaciones Geográficas, v. 53, p. 3-24, 2017. Doi: 10.5354/0719-5370.2017.41739.

SANCHES, A. M. Variações na extensão da cobertura de gelo do Nevado Cololo, Bolívia. Dissertação. (Mestrado em Geociências). Universidade Federal do Rio Grande do Sul. 93p. 2013. Disponível em: 〈https://lume.ufrgs.br/handle/10183/72104>.

SCHAEFER, M. et al. Modeling past and future surface mass balance of the Northern Patagonia Icefield. Journal of Geophysical Research. 118. p. 571-88. 2013. Doi: 10.1002/jgrf.20038.

VANDEKERKHOVE, E. The volcanic ash soils of Northern Chilean Patagonia $\left(44^{\circ}-48^{\circ} \mathrm{S}\right)$ : Distribution, weathering and influence on river chemistry. Universiteit Gent, Faculteit Wetenschappen: Gent. 99p. 2014. Disponível em: <https://lib.ugent.be/fulltxt/RUG01/002/163/638/RUG01002163638_2014_0001_AC.pdf>. 